\title{
Few young children had mild reactions to the measles, mumps, and rubella vaccine and older children had nearly no vaccine related reactions
}

Virtanen M, Peltola H, Paunio M, et al. Day-to-day reactogenicity and the healthy vaccinee effect of measles-mumps-rubella vaccination. Pediatrics 2000;106:e62.

\section{QUESTION: What are the adverse effects of the measles, mumps, and rubella (MMR) vaccine in children who are twins?}

\section{Design}

Randomised (unclear allocation concealment), blinded (nurses, investigators, and parents), placebo controlled, crossover trial.

\section{Setting}

A child health centre in Finland.

\section{Patients}

1162 children who were monozygous or heterozygous twins (581 twin pairs) between 14 months and 6 years of age with or without previous measles vaccination. Follow up was $100 \%$.

\section{Intervention}

For each twin pair, 1 child was allocated to receive a dose of vaccine first and then a dose of placebo, and the other child was allocated to receive a dose of placebo first and then a dose of vaccine given 3 weeks apart.

\section{Main outcome measures}

For 21 days, parents reported daily signs and symptoms including local reactions (redness with a diameter $>1$ inch, soreness, and swelling), rectal temperature, rhinorrhoea or cough, nausea or vomiting, diarrhoea, rash, arthralgia, conjunctivitis, staying in bed, drowsiness, and irritability.

\section{Main results}

Overall, $6 \%$ of the children had vaccination related events. During the first 2 days after injection, $4 \%$ of children had local reactions regardless of whether vaccine or placebo was given. Among children 14-18 months of age, systemic events developed between 6 and 14 days after injection with a peak on day 10 after both the first and second injection, regardless of order. Those who received the vaccine had a greater risk of MMR related events (table). Among children 6 years of age, arthralgia was the only symptom that was associated with vaccination. Zygocity, sex, history of allergy, and infections were not related to vaccination.

Odds ratios (ORs) for measles, mumps, and rubella (MMR) vaccine related events in children 14-18 months of age

Source of funding: Merck Research Laboratories.

For correspondence: Dr H Peltola, Helsinki University Central Hospital, Hospital for Children and Adolescents, Box 281 o0029 HUS, Helsinki Finland.Fax +3589 47174708 .

\section{MMR vaccine related events OR $(95 \% \mathrm{Cl}) \quad \mathrm{p}$ Value}

\begin{tabular}{lcc} 
Any MMR related event & $1.62(1.30$ to 2.03$)$ & $<0.001$ \\
\hline Fever $\geqslant 38.5{ }^{\circ} \mathrm{C}$ & $3.28(2.23$ to 4.82$)$ & $<0.001$ \\
\hline Respiratory symptoms & $2.66(1.66$ to 3.08$)$ & $<0.05$ \\
\hline Rash & $1.77(1.27$ to 2.47$)$ & $<0.001$ \\
\hline Arthralgia & $3.66(1.74$ to 7.70$)$ & $<0.001$ \\
\hline Conjunctivitis & $2.49(1.59$ to 3.90$)$ & $<0.001$ \\
\hline Staying in bed & $1.83(1.10$ to 3.03$)$ & 0.02 \\
\hline Irritability & $1.60(1.19$ to 2.16$)$ & $<0.002$ \\
\hline
\end{tabular}

\section{Conclusions}

In children who are twins between 14 and 18 months of age, few had mild reactions during the first 2 weeks after injection of the measles, mumps, and rubella vaccination. Children aged 6 years had almost no reaction to the vaccination.

\section{COMMENTARY}

Randomised controlled trials on vaccine reactogenicity are rare but important because the more common and easily done uncontrolled studies tend to exaggerate findings. This trial by Virtanen $e t$ al is well designed. Participants were twins, which maximised the similarity between comparison groups. The public health nurses who gave the injections and the parents who recorded the signs and symptoms were blinded to whether the children received the vaccine or placebo. The investigators used the MMR vaccine that is in the widest use internationally, which increases the generalisability of the findings. The study was partially funded by the company that produced the vaccine and, in such cases, readers should always consider potential biases that could arise.

The study had various interesting findings. Firstly, only $6 \%$ of children experienced adverse events attributable to the MMR vaccine, which the authors describe as "dramatically less common" than suggested by previous uncontrolled studies; secondly, the consistency between the timing of the signs and symptoms (beginning 5-7 d after vaccination and peaking on day 10) and the usual incubation period of measles (8-12 d) shows that they were primarily caused by the measles component of the vaccine; thirdly, a few young children experienced fever, rash, arthralgia, conjunctivitis, irritability, and the need to stay in bed as a result of the MMR; fourthly, other than arthralgia, the MMR vaccine did not cause reactions when given at 6 years of age, most likely because $>95 \%$ of children had already received measles vaccination or experienced the disease; and fifthly, respiratory symptoms and signs increased in frequency by $15 \%-20 \%$ during the first 10 days after injection in children who received either vaccine or placebo, suggesting that they were not attributable to MMR vaccination but more likely related to common infections.

Practitioners have an important part to play in educating parents of children about immunisation and potential local and systemic reactions. Those involved in immunisation programmes that include a 2 dose measles immunisation schedule can inform parents that the second dose is likely to result in fewer and less severe reactions, as long as the interval between doses does not exceed 5 years. Finally, reinforcing the reporting of severe local and systemic reactions to local health authorities is critical to improving the data base on immunisation reactions.

Mary Anne Carson, RN, BScN Health Manager, Communicable Disease Control Halton Regional Health Department Oakville, Ontario, Canada 